\title{
Does Laparoscopic Splenectomy have the Advantage on Postoperative Pain?
}

\author{
Jae Ryong Shim, M.D., Sung Pil Yun, M.D., Hyung-II Se0, M.D., Ph.D. \\ Department of Surgery, Biomedical Research Institute, Pusan National University Hospital, Busan, Korea
}

Purpose: The aim of this study is to elucidate the fundamental characteristics of the laparoscopic
splenectomy and to compare the clinical outcomes and postoperative pain between the laparoscopic
splenectomy and the conventional open splenectomy.

Methods: From January 2005 to January 2013, 28 patients underwent a splenectomy at Pusan National University Hospital, South Korea (PNUH). This study was a comparison of the demographic features and clinical results between the laparoscopic splenectomy $(\mathrm{n}=15)$ and open splenectomy $(n=13)$.

Results: For the two groups of patients, the following were similar: estimated blood loss, transfusion, operative time, duration of patient-controlled analgesia, and the additional administration of painkillers. In the laparoscopic splenectomy group, the postoperative hospital stay ( $7.9 \pm 1.6$ days versus $5.9 \pm 1.4$ days, $p=0.002)$ and the diet start time ( $2.7 \pm 0.3$ days versus $1.8 \pm 0.8$ days, $p=0.003$ ) were significantly shorter. No significant difference in postoperative pain was observed between the two groups.

Conclusion: In this study, there was no benefit for postoperative pain in the LS group. However, the laparoscopic splenectomy has several benefits, including a shorter postoperative hospital stay and an earlier diet start time; in addition, it is feasible and safe.

Keywords: Laparoscopic splenectomy, Open splenectomy, Clinical outcomes

This is an Open Access article distributed under the terms of the Creative Commons Attribution Non-Commercial License (http:// creativecommons.org/licenses/by-nc/4.0/) which permits unrestricted non-commercial use, distribution, and reproduction in any medium, provided the original work is properly cited.
Received June 9, 2015

Revised 1st July 12, 2015

2nd August 12, 2015

3rd August 17, 2015

Accepted August 18, 2015

Corresponding author

Hyung-Il Seo

Department of Surgery, Biomedical Research Institute, Pusan National University Hospital, 189 Gudeok-ro, Seo-gu, Busan 49241, Korea Tel: +82-51-240-7238

Fax: +82-51-247-1365

E-mail: seohi71@hanmail.net
Copyright (๐) 2015 The Journal of Minimally Invasive Surgery. All rights reserved.

\section{INTRODUCTION}

Since the first laparoscopic surgery (LS) was performed and reported by Delaitre in 1991, the LS has now become the gold standard of the surgical management of benign and normal-sized spleen, such as those found in idiopathic thrombocytopenic purpura (ITP)., ${ }^{1,2}$ Several studies have confirmed the advantage of LS over the open splenectomy (OS), including decreased blood loss, a shorter hospital stay, and more rapid recovery. ${ }^{3-5}$ Moreover, the LS is safely done on a patient with splenomegaly because of the development of laparoscopic techniques. However, the ITP might be a challenge for an inexperienced surgeon. This study aimed to elucidate the fundamental characteristics of the laparoscopic splenectomy and to compare the clinical outcomes and postoperative pain between the laparoscopic splenectomy and conventional open splenectomy. Furthermore, this study is based on the authors' experience with LS.

\section{MATERIALS AND METHODS}

Patients were enrolled in this study who underwent a splenectomy at the Pusan National University Hospital (PNUH) from January 2005 to January 2013. A retrospective review 
was performed based on the medical records. In this study, LS was generally performed since 2007. OS was performed in every case before 2007. In the cases of OS, surgeries were performed by several operators before 2007, and LS was performed by single operator since 2007. Operative indication includes the case of normal size spleen, low risk of malignant potency and that hematologic malignancy is in a spleen parenchyma. Although there are several operators who performed OS cases, according to medical reports, there are no significant differences about approaching methods or order of splenectomy. Five cases of OS were performed by single operator after 2007. In that case, there were 3 lymphoma, 1 hereditary spherocytosis with splenomegaly, and 1 ITP. The splenectomy for trauma and pediatric patients were not included in this study. In total, 28 patients were included. For the comparison between the LS and OS, the records of 28 patients, undergoing a splenectomy, were evaluated for age, gender, operation time, EBL, time to start of diet, hospital stay, spleen size, transfusion, patient-controlled analgesia (PCA) duration, and use of an additional pain killer such as opioid and nonsteroidal anti-inflammatory drugs (NSAIDs). Opioids painkiller such as fentanyl patch and pethidine was used when patient complain of pain. The visual analogue scale (VAS) and numeric rating scale (NRS) for assessment of pain are well-known and are equally sensitive in assessing acute pain after surgery. ${ }^{6}$ We used NRS for assessment of pain (Fig. 1). The pain with over NRS 6, opioid painkiller was taken. And NSAIDs was taken when over NRS 4. Fentanyl patch 25 mcg was calculated to equivalent intravenous pethidine $150 \mathrm{mg}$ by NCCN guideline?

The LS surgeries were performed under general anesthesia and supine position with right sided tilting. The surgeon stood on the patient's right side, with the first assistant on the patient's left side, and the camera assistant stood to the surgeon's right. The camera port $(12 \mathrm{~mm})$ was placed below the umbilicus. One 12-mm port which performed using a harmonic scalpel (Ethicon Endo-Surgery Inc., Cincinnati, $\mathrm{OH}, \mathrm{USA}$ ) and then an Endo GIA (Covidien Corp., Dublin, Ireland) was then inserted about $4 \sim 5 \mathrm{~cm}$ below the costal margin along the left midclavicular line. One 5-mm assistant port was placed in the subxiphoid area and the other along the left anterior axillary line. Without lift of posterior wall of

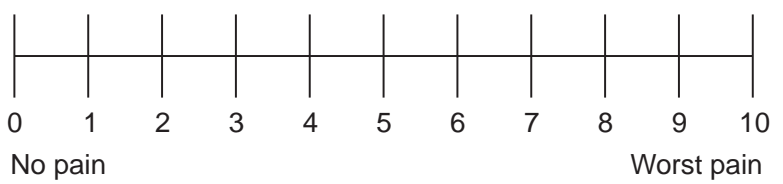

Fig. $1.0 \sim 10$ numeric rating scale (NRS). stomach, we approached splenic hilum first, and vessels were ligated. Splenic artery and vein is ligated individually by metal or weck clip. And then short gastric vessels were ligated. Next, splenocolic mobilization was performed. After the laparoscopic procedure, the spleen is extracted by morcellation within a sterile plastic bag, and removed through the umbilical incision. Endovascular GIA white cartilage (Covidien Corp., Dublin, Ireland) was used to ligate a splenic vein in 2 patients who have thick vein that is impossible to ligate with clip. And each splenic artery and vein was ligated separately in every patient. In the case of OS, the operation was performed with an upper midline incision.

Next, the clinical outcomes between LS and OS were analyzed. The categorical variables were compared by the Chi-square test or Fisher's exact test. Continuous variables were compared by $t$-test and the Mann-Whitney U-test. The $p$ values less than 0.05 were considered statistically significant.

\section{RESULTS}

Hemato-oncological disease was the most common in the elective splenectomy, and the LS were performed most often in the ITP. The indications for LS were ITP in 10 patients, lymphangioma in 2 patients, idiopathic portal hypertension, splenic cyst, diffuse B cell lymphoma patients. The LS was successfully performed in all patients. There was no conversion to open surgery, as well as no postoperative complications. Thirteen patients were operated on using the open procedure: 3 ITP, 4 hereditary spherocytosis, 4 lymphoma, 1 CML, 1 hereditary hemolytic anemia (Table 1). The clinical features between LS and OS are summarized in Table 2. Of the 28 patients, 15 patients underwent the LS. Of these, 12 were female and 3 were male. The mean age was

Table 1. The indication for laparoscopic splenectomy versus open splenectomy

\begin{tabular}{lcc}
\hline & LS $(\mathbf{n}=15)$ & OS ( $=13)$ \\
\hline Hemato-oncologic disease & 10 & 3 \\
ITP & 0 & 4 \\
Hereditary spherocytosis & 1 & 4 \\
Lymphoma & 0 & 1 \\
CML & & \\
Benign disease & 2 & 0 \\
$\quad$ Lymphangioma & 1 & 1 \\
$\quad$ Splenic cyst & 1 & 0 \\
$\quad$ Idiopathic portal hypertension & & \\
ITP = Idiopathic thrombocytopenic purpura; CML = Chronic myelocytic \\
leukemia.
\end{tabular}


Table 2. Comparison of characteristics between laparoscopic splenectomy and open splenectomy

\begin{tabular}{lccc}
\hline & LS ( $\mathbf{n}=15)$ & OS $(\mathbf{n}=13)$ & $p$ value \\
\hline Sex & & & \\
$\quad$ Female/male & $12 / 3$ & $7 / 6$ & 0.228 \\
Age (year) & $43.6 \pm 10.9$ & $41.0 \pm 11.4$ & 0.543 \\
Operation times (min) & $219.0 \pm 77.0$ & $190.0 \pm 68.3$ & 0.305 \\
EBL (ml) & $390.0 \pm 464.5$ & $992.3 \pm 1,162.2$ & 0.076 \\
Time to start of diet (day) & $1.8 \pm 0.8$ & $2.7 \pm 0.3$ & 0.003 \\
Hospital stay (day) & $5.9 \pm 1.4$ & $7.9 \pm 1.6$ & 0.002 \\
Spleen size (cm) & $12.1 \pm 4.4$ & $16.6 \pm 5.5$ & 0.023 \\
RBC Transfusion (n) & 3 & 6 & 0.228
\end{tabular}

$\mathrm{EBL}=$ estimated blood loss; PCA = patient-controlled analgesia; NSAID $=$ nonsteroidal anti-inflammatory drugs.

$43.6 \pm 10.9$ years and the ages ranged from 25 and 61 years. Of the 13 patients undergoing OS, 7 were female and 6 were male. The mean age was $41.0 \pm 11.4$. There was no significant difference concerning gender and age between the two groups. Furthermore, there was no significant difference between two groups regarding: operative time, estimated blood loss, transfusion (Table 2). And there was no significant difference about postoperative pain such as PCA duration, usage of an additional painkiller, and total usage dose of opioid and NSAIDs. But frequency of opioid painkiller was higher in OS patients. (Table 3) In patients who underwent the LS, the mean hospital stay was $5.9 \pm 1.4$ days, and the time to the start of diet was $1.8 \pm 0.8$ days, and the spleen size was $12.1 \pm 4.4 \mathrm{~cm}$. In the OS, mean hospital stay and time to start of diet were $7.9 \pm 1.6$ days and $2.7 \pm 0.3$ days, and spleen size was $16.6 \pm 5.5$ $\mathrm{cm}$. The time to the start of the diet $(p=0.003)$ and hospital stay $(p=0.002)$ was shorter in the LS group, and the spleen size was larger in OS group ( $p=0.023)$ (Table 2). There was only one complication, which was postoperative bleeding in the OS group; and it was controlled by angiographic embolization, not by re-operation.

\section{DISCUSSION}

Since Delaitre successfully performed the first laparoscopic splenectomy in 1991, the LS has now become accepted as the gold standard of surgical management in ITP. In the case of a non-splenomegaly patient, it is suitable to operate with laparoscopic surgery, but in case of hereditary spherocytosis or hereditary hemolytic anemia, splenomegaly is quite common, thus it is difficult to perform laparoscopic surgery. ${ }^{8}$ Furthermore, laparoscopic surgery is not commonly performed
Table 3. Comparison of postoperative pain between laparoscopic splenectomy and open splenectomy

\begin{tabular}{lccc} 
& $\mathrm{LS}(\mathrm{n}=15)$ & $\mathrm{OS}(\mathbf{n}=13)$ & $p$ value \\
\hline PCA duration (day) & $3.1 \pm 1.0$ & $2.3 \pm 1.5$ & 0.117 \\
Using additional pain killer (n) & 9 & 11 & 0.221 \\
Opioid (n) & 6 & 11 & 0.024 \\
Opioid $(\mathrm{mg})$ & $50.0 \pm 115.7$ & $173.0 \pm 304.4$ & 0.158 \\
NSAIDs (n) & 6 & 7 & 0.705 \\
NSAIDs (mg) & $22.0 \pm 40.0$ & $46.2 \pm 60.8$ & 0.220 \\
\hline
\end{tabular}

PCA = patient-controlled analgesia; NSAID = nonsteroidal anti-inflammatory drugs.

in patients who have primary splenic malignancy or splenic metastasis, because of the possibility of seeding metastasis during operation. Recently, laparoscopic surgery in malignant patients and those with splenomegaly were performed because of the development of laparoscopic equipment and techniques, however there has not been a thorough meta-analysis of the laparoscopic surgery on those kind of patients. In present study, OS were performed by several operators before 2007, and LS was performed by single operator since 2007. Until recently, we usually did not perform LS in the patients with malignancy or metastasis for reasons mentioned above.

As can be ascertained from previous studies, there are many advantages in the laparoscopic splenectomy, such as less perioperative morbidity, a shorter hospital stay, less postoperative pain, better cosmesis, and a shorter time to the start of diet. Winslow et al. ${ }^{5}$ reported LS is associated with a significant reduction in the incidence of postoperative complication compared with OS. Bulus et al. ${ }^{9}$ described that LS is superior with regard to hospital stay, start time to diet and cosmesis. Brunt et al. ${ }^{4}$ reported that patients who underwent LS required significantly less parenteral pain medications. In this study, the postoperative hospital stay $(7.9 \pm 1.6$ days versus $5.9 \pm 1.4$ days, $p=0.002)$ and diet start time (1.8 \pm 0.8 days versus $2.7 \pm$ 0.3 day, $p=0.003$ ) was significantly shorter in the laparoscopic splenectomy group. And in patients who underwent the OS, the PCA duration and a usage of additional painkiller were higher than the LS group, but those were not statistically significant. As previously stated, one advantage of LS is less postoperative pain. But in this study, our result shows that there is no significant advantage about postoperative pain in LS. And it is considered that because of a protocol of PCA in PNUH. The PCA is a routine procedure in the entire patients who undergo operation at PNUH.

Nowadays, the LS is a standard procedure for the surgical treatment of hematologic disorders, such as ITP and thrombotic 
thrombocytopenic purpura. ${ }^{2}$ Furthermore, Burch et al., ${ }^{10}$ reported that malignant splenic disease is not a contraindication of LS, because there are no differences of morbidity and mortality between patients who have benign disease that underwent LS, versus those who were malignant, and the LS did not affect long-term survival. In this study, the LS were performed in patients with a benign disease, such as ITP, in 10 cases, whereas OS was performed in patients with a malignant disease such as lymphoma. In present study, there was only one case of lymphoma, and the patient is on observation without any recurrence. But we submit that more study is needed about lymphoma. Recently, LS is frequently performed in malignant cases, such as lymphoma or splenic metastasis that it is not included in this study.

In the case of splenomegaly, the LS is a relative contraindication because of its difficult technique. The LS has also a greater morbidity and conversion rates in larger spleens for both experienced and inexperienced surgeons. ${ }^{2,11,12}$ Furthermore, splenomegaly makes it difficult to secure a view of the splenic hilum, and easily leads to open conversion or postoperative complications owing to massive bleeding or capsule injury, and also because of severe adhesion to adjacent organs. ${ }^{8}$ In this study, the spleen size of LS is $12.1 \pm 4.4 \mathrm{~cm}$, and for OS, it is $16.6 \pm 5.5 \mathrm{~cm}$, which is statistically significant. But in a recent case, the LS was successfully performed on a patient with a 1,500 cc spleen, so it is expected that LS will be performed in patients who have splenomegaly.

In this study, for LS, there is a statistically significant advantage in a shorter hospital stay and diet start time. We usually start the sipping on clear fluids on the postoperative day (POD) 1 morning, and a soft diet is started on the POD 1 afternoon; and if there is no complication with the diet, this diet is adhered to until the discharge day. The postoperative hospital stay was statistically significantly short in the case of the LS group (7.9 \pm 1.6 days versus $5.9 \pm 1.4$ days, $p=0.002$ ). There is no difference between the postoperative pain in the LS and OS groups; however, we conjecture that the OS group will have a longer hospital stay because of the longer time for the main wound care, as well as the late diet start time.

In conclusion, the LS is a safe and useful technique, and it can be expected that inexperienced surgeons can successfully perform it because of the development of laparoscopic technique and equipment. In this study, it is patently clear that the LS is better than OS for hospital stay, diet start time, and cosmesis. Recently, the LS was performed in patients who have splenomegaly or massive splenomegaly, and in wellselected cases, LS is no longer a contraindication. However, prospective research is certainly needed.

\section{ACKNOWLEDGEMENTS}

This work was supported by clinical research grant from Pusan National University Hospital 2015.

\section{REFERENCES}

1) Delaitre B, Maignien B. Splenectomy by the laparoscopic approach. Report of a case. Presse medicale (Paris, France: 1983) 1990;20:22632263.

2) Sharma D, Shukla VK. Laparoscopic splenectomy: 16 years since Delaitre with review of current literature. Surgical Laparoscopy Endoscopy \& Percutaneous Techniques 2009;19:190-194.

3) Park A, Marcaccio M, Sternbach M, et al. Laparoscopic vs open splenectomy. Archives of Surgery 1999;134:1263-1269.

4) Brunt LM, Langer JC, Quasebarth MA, et al. Comparative analysis of laparoscopic versus open splenectomy. The American journal of surgery 1996;172:596-601.

5) Winslow ER, Brunt LM. Perioperative outcomes of laparoscopic versus open splenectomy: a meta-analysis with an emphasis on complications. Surgery 2003;134:647-653.

6) Breivik H, Borchgrevink PC, Allen SM, et al. Assessment of pain. Br J Anaesth 2008;101:17-24.

7) Swarm RA, Abernethy AP, Anghelescu DL, et al. Adult cancer pain. Journal of the National Comprehensive Cancer Network 2013;11:992-1022.

8) Rosen M, Brody F, Walsh RM, et al. Hand-assisted laparoscopic splenectomy vs conventional laparoscopic splenectomy in cases of splenomegaly. Archives of Surgery 2002;137:1348-1352.

9) Bulus H, Mahmoud H, Altun H, et al. Outcomes of laparoscopic versus open splenectomy. J Korean Surg Soc 2013;84:38-42.

10) Burch M, Misra M, Phillips EH. Splenic malignancy: a minimally invasive approach. The Cancer Journal 2005;11:36-42.

11) Kercher KW, Matthews BD, Walsh RM, et al. Laparoscopic splenectomy for massive splenomegaly. The American journal of surgery 2002;183:192-196.

12) Patel AG, Parker JE, Wallwork B, et al. Massive splenomegaly is associated with significant morbidity after laparoscopic splenectomy. Annals of surgery 2003;238:235. 